\title{
Molecular and Cellular Aspects of Atherosclerosis: Emerging Roles of TRPC Channels
}

\author{
Guillermo Vazquez, Kathryn Smedlund, \\ Jean-Yves K. Tano and Robert Lee \\ Department of Physiology and Pharmacology, \\ University of Toledo Health Science Campus, Toledo, $\mathrm{OH}$, \\ USA
}

\section{Introduction}

\subsection{Endothelial inflammatory signaling and monocyte recruitment}

Recruitment of circulating monocytes to activated areas of the endothelium and their migration to the subintimal inflammatory foci represents one of the earliest events in atherogenesis (Linton and Fazio 2003; Hansson 2005). Importantly, monocyte recruitment can be recognized throughout all lesional stages including advanced lesions, where plaque infiltration and neovascularization occur. Indeed, available experimental evidence supports the notion that in advanced stages monocyte infiltration contributes to plaque instability and rupture (Virmani, Burke et al. 2006). Monocyte recruitment to the subendothelial milieu implies a sequence of events that begin with monocyte rolling along and tethering to the endothelial surface, firm adhesion and activation, and ultimately migration to the subintima. At the molecular level, the entire sequence entails interaction of integrins on the monocyte surface with cell adhesion molecules (CAMs) expressed on the endothelial cell. Monocyte rolling and tethering is mainly mediated by CAMs from the selectin group (v.g., E-selectin) while firm adhesion and migration are mostly mediated by CAMs from the immunoglobulin (Ig) superfamily, such as intercellular cell adhesion molecule-1 (ICAM-1) and vascular cell adhesion molecule-1 (VCAM-1). Compelling evidence accumulated over the last decade has clearly established that VCAM-1 (CD106) has a prominent role in mediating attachment and migration of monocytes (the Cluster of Differentiation nomenclature (Zola, Swart et al. 2005) is given for reference, but "VCAM-1" will be used throughout the text).

\subsubsection{VCAM-1 expression and atherosclerosis}

Although other adhesion molecules, such as ICAM-1 (CD54) or E-selectin, also contribute to adhesion of monocytes to endothelial cells, VCAM-1 is unique in that its expression level and pattern are highly sensitive to the action of several pro-inflammatory/proatherogenic stimuli. While other CAMs are constitutively expressed in non-activated 
endothelium, VCAM-1 is virtually absent, but its markedly upregulated when endothelium is exposed to atherorelevant stimuli (Galkina and Ley 2007). For example, in hypercholesterolemic animals both ICAM-1 and VCAM-1 are induced in early lesions. However, VCAM-1 expression is largely restricted to lesions, or to sites prone to lesion formation, and can also be detected even before the onset of visible fatty streaks, while ICAM-1 also extends into uninvolved aorta and lesion-protected regions (Iiyama, Hajra et al. 1999). This dissimilar pattern immediately suggested different functions for ICAM-1 and VCAM-1, at least in lesion initiation. A more direct comparison of VCAM-1 and ICAM-1 in atherosclerosis was possible through generation of mice homozygous for a VCAM-1 molecule lacking the Ig-like extracellular domain 4 (Vcam1D4D/D4D) which partially circumvents the embryonic lethality of $V$ cam-1\% mice. Using such mouse model it was possible to show that whereas both ICAM-1 and VCAM-1 are upregulated in lesions, VCAM-1 has a dominant role in early lesion formation (Cybulsky, Iiyama et al. 2001; Dansky, Barlow et al. 2001). Atherorelevant stimuli such as tumor necrosis factor-a (TNFa), locally released nucleotides, vascular endothelial growth factor (VEGF) or oxidized low density lipoprotein (oxLDL), are potent inducers of VCAM-1 expression (Galkina and Ley 2007). Depending on the stimulus and/or the regional location of the endothelial cell along the vascular bed, VCAM-1 expression is regulated through Nuclear Factor карраВ (NFкB), Nuclear Factor of Activated T cells (NFAT) or both (Armesilla, Lorenzo et al. 1999; Kim, Moon et al. 2001; Yao and Duh 2004).

\subsubsection{VCAM-1 structure and function}

Human VCAM-1 is a single transmembrane protein with seven Ig-like extracellular domains (1-7) but can also be expressed as a form with only domains 1-3 and 5-7 (Chuluyan, Osborn et al. 1995). The extracellular domains 1 and 4 mediate specific binding to the integrin $\alpha_{4} \beta_{1}$ (Very Late Antigen 4, VLA-4) on the monocyte facilitating firm adhesion to the endothelium. The cytoplasmic domain is only 19 amino acids long, and has a PDZ-binding motif, but specific interactions or functional relevance remain unknown. Interaction of VCAM- 1 with $\alpha_{4} \beta_{1}$ conveys a conformational message through the transmembrane domain towards the cytosolic region, triggering intracellular signaling events. Importantly, VCAM-1-dependent signaling is a prerequisite for successful migration of bound monocytes (Matheny, Deem et al. 2000; Deem, Abdala-Valencia et al. 2007). Some salient features within this signaling are the recruitment and activation of the Rho-family GTPase Rac-1, Rac-1 dependent stimulation of NADPH oxidase and production of hydrogen peroxide $\left(\mathrm{H}_{2} \mathrm{O}_{2}\right)$ (van Wetering, van den Berk et al. 2003). VCAM1-dependent release of $\mathrm{H}_{2} \mathrm{O}_{2}$ to the extracellular milieu is thought to contribute to activation of matrix metalloproteases and the increased endothelial permeability observed during atherogenesis. This may be particularly important in advanced lesions by creating additional endothelial damage and plaque instability (Virmani, Burke et al. 2006). Calcium $\left(\mathrm{Ca}^{2+}\right)$ release and influx subsequent to VCAM-1- $\mathrm{a}_{4} \beta_{1}$ interaction or antibodyinduced VCAM-1 crosslinking have also been related to the signaling required for monocyte migration (Isabelle Ricard 1997; Cook-Mills 2002; Cook-Mills, Johnson et al. 2004). However, neither the nature of the channels involved nor the underlying mechanism/s are yet known. In coronary artery endothelial cells of human origin (HCAECs) activation of VCAM-1 by antibody-induced crosslinking results in 
approximately three-fold increase in the rate of cation influx compared to non-treated cells (Smedlund and Vazquez, unpublished observations). Such increase in cation influx occurs over the existing constitutive influx, and thus is possible that both constitutive and regulated activity of cation channels play a role. Because TRPC 3 accounts for most of the constitutive cation entry in HCAECs and also significantly contributes to regulated influx (Vazquez and Putney 2006; Smedlund and Vazquez 2008) it is likely that TRPC3 represents a component of the signaling underlying VCAM-1-dependent monocyte transmigration.

\subsection{Role of macrophage survival and apoptosis in lesion development}

As mentioned above, monocyte recruitment to the subintima is a fundamental event in atherogenesis (Linton and Fazio 2003). Transmigration of the monocyte to the subendothelial milieu is followed by its differentiation into macrophage, which is now recognized as a key cell component in determining lesion progression and fate. Lesional macrophages engulf modified lipoproteins, mostly oxLDL, becoming lipid-laden macrophages; this results in a lipid overload of the macrophage which imposes a significant stress to the endoplasmic reticulum (ER), mostly due to accumulation of free cholesterol. Consequently, an irreversible ER-stress response is triggered leading to macrophage apoptosis. Indeed, the majority of apoptotic lesional cells are macrophages (Linton and Fazio 2003; Tabas 2010). Clearance of apoptotic cells is conducted by resident phagocytes, which phagocytose the apoptotic macrophage and exit the lesion site through lymphatic circulation or by migrating back to the blood stream. This clearing process, known as efferocytosis, is crucial in preventing the apoptotic cells from dying in situ which would otherwise lead to post-apoptotic necrosis and the subsequent exacerbation of the inflammatory response. Notably, the balance between production of apoptotic macrophages and their clearance by efferocytosis constitutes a defining factor in lesion formation, remodeling and progression ((Tabas 2010) and references therein). For instance, macrophage apoptosis in early lesions is beneficial in that reduces lesion cellularity and size and plaque progression (Arai, Shelton et al. 2005; Liu, Thewke et al. 2005; Babaev, Chew et al. 2008; Wang, Liu et al. 2008) while increased apoptosis in advanced plaques enlarges the necrotic core and promotes plaque instability (Linton, Babaev et al. 1999; Seimon, Wang et al. 2009; Yancey, Blakemore et al. 2010). Thus, altered expression and/or deregulation of signaling proteins directly or indirectly involved in macrophage survival/apoptosis can have a significant impact on lesion progression.

\section{TRPC channels}

\subsection{Structure, function and role in cardiovascular disease}

Calcium influx has long been recognized as an essential component of physiological and pathophysiological events. Changes in intracellular $\mathrm{Ca}^{2+}$ concentration that follow $\mathrm{Ca}^{2+}$ influx through plasma membrane $\mathrm{Ca}^{2+}$ channels not only modulate a myriad of $\mathrm{Ca}^{2+}$ dependent signaling pathways but also affect the driving force for other ions by modifying the membrane potential. $\mathrm{Ca}^{2+}$ influx is of particular importance in vascular function and cardiovascular disease, where the effects of $\mathrm{Ca}^{2+}$ influx can be seen throughout the entire cardiovascular system, in smooth muscle and endothelial cells, cardiomyocytes, 
lymphocytes, monocytes, macrophages, among other cell types. Of the many $\mathrm{Ca}^{2+}$ channels identified in the last half century, Transient Receptor Potential Canonical (TRPC) channels are recognized as major contributors to $\mathrm{Ca}^{2+}$ influx and play a role in various physiological and pathological states. The TRPC family belongs to the TRP superfamily of ion channel forming proteins, and are the most closely related to the founding member Drosophila TRP protein (Vazquez, Wedel et al. 2004). TRPC proteins can be grouped into four subgroups, TRPC1, TRPC2 (a pseudogene in humans), TRPC3/6/7 and TRPC4/5 (Trebak, Vazquez et al. 2003; Vazquez, Wedel et al. 2004).

Despite some structural variation across the subgroups of the TRPC family, there are several structural motifs which are conserved throughout members. The cytoplasmic Nand C-termini are separated by six transmembrane domains (TM1-TM6), with a re-entry loop between TM5 and TM6 which is thought to line the wall of the channel pore ((Vazquez, Wedel et al. 2004) and references therein). Other shared structural motifs of TRPCs include ankyrin repeats and a putative caveolin binding site on the N-terminus, and on the C-terminus the so called TRP signature motif (EWKFAR), a proline-rich motif and a calmodulin $/ \mathrm{IP}_{3}$ receptor binding (CIRB) site; predicted coiled-coil regions are present on both N- and C-termini, and in TRPC4 and 5, an extended C-terminus includes a PDZ binding motif (Vazquez, Wedel et al. 2004). An examination of the function of the TRPC cytoplasmic motifs hints at mechanisms of channel activation and signaling pathways. For instance, ankyrin repeats form specialized structures with the repeated units stacking against one another to form a protein-binding interface; this allows for interaction with other proteins and seems to play a role in channel trafficking to the plasma membrane. Coiled-coil regions are commonly associated with oligomerization and may contribute to formation of specific homo- and heterotetramers of TRPCs or association with other proteins containing coiled-coil motifs (Vazquez, Wedel et al. 2004). The proline-rich motif and CIRB region have also been associated to interactions with different signaling molecules, with variations existing throughout individual TRPC members. The mechanisms underlying activation and regulation of TRPC channels has been matter of extensive research and debate, with efforts mostly centered at elucidating whether they form store-operated (activated by mere depletion of internal $\mathrm{Ca}^{2+}$ stores) or non-store-operated channels (discussed in (Trebak, Vazquez et al. 2003; Vazquez, Wedel et al. 2004; Smyth, DeHaven et al. 2006). Whereas some properties of TRPC channels observed in heterologus expression systems correlate well with those of TRPCs expressed under native conditions, many others do not. It is imperative to elucidate the mechanism/s underlying regulation of TRPC channels in their native environment, as this would greatly contribute to assign definitive roles to individual TRPC members. Nevertheless, equally important to comprehend their role in cardiovascular physiology and disease is to identify cellular and molecular events which, directly or indirectly, may rely upon appropriate TRPC function.

TRPC proteins are ubiquitously expressed throughout the cardiovascular system and hematopoietic cells and all members have been implicated not only in physiological cardiovascular functions but most importantly, in the pathogenesis of cardiovascular disease. Indeed, TRPCs have been implicated in a variety of processes known to be critical in cardiovascular pathology such as endothelial dysfunction, vascular relaxation, oxidative 
stress, and angiogenesis among others. This has recently been reviewed by us (Tano, Smedlund et al. 2010) and others (Abramowitz and Birnbaumer 2009) and the reader is referred to those for further details. The following sections focus on recent findings that specifically point to a potential role of TRPC 3 in atherorelevant processes.

\section{Participation of TRPC3 in atherorelevant molecular/cellular processes}

\subsection{TRPC3 and regulated expression of VCAM-1}

Calcium signaling is an important component of the mechanism by which several inflammatory factors induce VCAM-1 expression. For instance, changes in intracellular $\mathrm{Ca}^{2+}$ associated to $\mathrm{Ca}^{2+}$ release from internal stores have been linked to the ability of Substance $\mathrm{P}$ to induce VCAM-1 subsequent to $\mathrm{Ca}^{2+}$-dependent activation of NFAT and NFKB in microvascular endothelium (Quinlan, Naik et al. 1999), and of $\beta_{2}$-microglobulin to induce VCAM-1 expression in synovial fibroblasts (Chen, O'Neill et al. 2002). In the human coronary endothelial cells HCAEC, $\mathrm{Ca}^{2+}$ mobilization contributes to lipoprotein A- and ATP-dependent VCAM-1 expression (Allen, Khan et al. 1998; Seye, Yu et al. 2003). Nevertheless, the specific role of $\mathrm{Ca}^{2+}$ influx in VCAM-1 expression was never directly examined before. In recent work we specifically explored the impact of $\mathrm{Ca}^{2+}$ influx in regulated expression of VCAM-1 in HCAECs (Vazquez and Putney 2006; Smedlund and Vazquez 2008). Our studies demonstrated that, whereas HCAECs express all members of the TRPC family, only TRPC3 forms, or is part of, endogenous $\mathrm{Ca}^{2+}$-permeable channels that contribute to ATP stimulated $\mathrm{Ca}^{2+}$ influx. Such mechanism occurs downstream ATPdependent activation of purinergic $\mathrm{P}_{2} \mathrm{Y}_{2}$ receptors and results in upregulation of VCAM-1 total and plasma membrane associated levels with subsequent increase in monocyte adhesion (Smedlund and Vazquez 2008). This represented the first direct indication that $\mathrm{Ca}^{2+}$ influx plays a role in the signaling driving VCAM-1 expression and that TRPC 3 forms native $\mathrm{Ca}^{2+}$-permeable channels whose activity is fundamental within the signaling underlying VCAM-1 expression and monocyte recruitment. Because TRPC3 is the only TRPC member whose high constitutive, non-regulated activity has been shown to operate under either heterologous or native expression conditions (Trebak, Vazquez et al. 2003) those findings raised the question whether TRPC3 contributes to expression of VCAM-1 through regulated activity, constitutive activity, or both. In a follow up study using TNFa to induce VCAM-1 expression and a combination of real-time fluorescence and silencing RNA approaches, we conclusively showed that it is the constitutive function of TRPC 3 which mediates most of the $\mathrm{Ca}^{2+}$ influx required for regulated expression of VCAM-1 in HCAECs (Smedlund and Vazquez 2008).

In most endothelial cells VCAM-1 expression is regulated, at the transcriptional level, by nuclear factor kappa B (NFkB)(Zhang 2008) and we have shown that this is also the case in HCAECs. NFkB activation involves its release from the inhibitory protein IkBa and then the subsequent translocation of $\mathrm{NFkB}$ to the nucleus where it modulates transcriptional activity of target genes. Release of NFkB from IkBa is preceded by phosphorylation of IkBa by IkBa kinases (IKKs) followed by its ubiquitination and proteasomal degradation (Tergaonkar 2006). Because in most cells types examined so far NFkB activation depends, directly or indirectly, on $\mathrm{Ca}^{2+}$ influx, and TNFa-induced VCAM-1 requires constitutive $\mathrm{Ca}^{2+}$ influx (Smedlund, Tano et al. 2010), we examined 
whether TRPC3, through its constitutive function, contributes to the mechanism by which NFkB modulates VCAM-1 expression in HCAECs. Interestingly, our studies showed that knockdown of TRPC3 in HCAECs drastically reduced the ability of TNFa to induce phosphorylation of IkBa and its upstream regulator IKK $\beta$ (Smedlund, Tano et al. 2010), and this correlated with an inhibition of IkBa degradation. These findings indicated for the first time that TRPC3 constitutive function is an obligatory component in the signaling driving TNFa-dependent activation of NFkB. In addition, we showed that TRPC3mediated $\mathrm{Ca}^{2+}$ entry is fundamental to activate the calmodulin (CAM)/calmodulin kinase II (CAMKII) axis in a NADPH oxidase-dependent manner, and this signaling axis in turn activates NFkB (Smedlund, Tano et al. 2010). Importantly, our studies brought about a conceptually novel perspective on the role of TRPCs in cardiovascular disease, as they underscored for the first time, the potential pathological impact of upregulated expression of a TRPC channel endowed with high constitutive activity and how this may relate with pathological $\mathrm{Ca}^{2+}$-dependent signaling, independently of the canonical pathway driven by receptor stimulation. This is of particular interest to the field, as in those instances where TRPCs participate in mechanisms associated to inflammatory vascular disease (reviewed by us in (Tano, Smedlund et al. 2010)) their contribution relates to regulated, or receptordependent channel function, rather than constitutive activity. In vivo studies are underway using mouse models of atherosclerosis with genetically manipulated levels of endothelial TRPC3 in order to determine the potential impact of TRPC3 expression and constitutive function in the context of the molecular and cellular events that lead to atherosclerotic lesion development in the intact vessel.

\subsection{TRPC3 and macrophage survival}

As stated earlier (section 2.2) the balance between apoptotic macrophages and their clearance by resident phagocytes at the lesion site is determinant in the progression of the atherosclerotic lesion. Within this context, signaling events that modulate the survival rate of the macrophage have a tremendous impact on such balance, provided efferocytic properties of resident phagocytes remain unaffected. Recent studies in our laboratory have implicated non-regulated, constitutive $\mathrm{Ca}^{2+}$ influx in the signaling associated with macrophage survival. Two major pathways are essential for the survival of macrophages in the atherosclerotic lesions: the phosphatidylinositol-3-kinase (PI3K)/ AKT axis and the NFkB route. In the PI3K/AKT pathway, macrophage survival signals (v.g., insulin-like growth factor, prostaglandin E2) acting through either receptor tyrosine kinases or G-protein coupled receptors induce activation of PI3K in the plasma membrane and the subsequent generation of 3'-phosphorylated phosphoinositides such as phosphatidylinositol 3, 4 bisphosphate/3,4,5 trisphosphate. These phosphoinositides allow for recruitment and activation of PDK-1 which then leads to full activation of AKT kinase. One of the major mechanisms of AKT-dependent survival takes place through AKT-mediated phosphorylation of the pro-apoptotic protein BAD, a member of the Bcl-2 family. Upon AKT-mediated phosphorylation, BAD releases the anti-apoptotic proteins Bcl-2 and Bcl-x, preventing mitochondrial release of cytochrome $\mathrm{c}$ and thus progression of the mitochondrial apoptotic pathway (Datta, Brunet et al. 1999). As for macrophage survival through the transcription factor NFKB, it is known that activation of NFkB exquisitely regulates the transcriptional status of several survival genes. Both of the survival pathways described 
above are highly active in THP-1 derived macrophages (TDMs) upon exposure to the atherorelevant cytokine TNFa (Tano and Vazquez 2011). Notably, maneuvers that prevent constitutive $\mathrm{Ca}^{2+}$ entry through $\mathrm{Ca}^{2+}$ permeable channels drastically reduce the phosphorylation of $\mathrm{IkBa}, \mathrm{AKT}$ and its downstream target BAD, with the subsequent increase in macrophage apoptotic rate (Tano and Vazquez 2011). In addition, when TDMs are pre-treated with pharmacological inhibitors of CAM and CAMKII, activation of survival signaling is prevented as efficiently as blockade of constitutive $\mathrm{Ca}^{2+}$ influx does. These findings indicated for the first time that activation of macrophage survival pathways depends, to a significant extent, on constitutive $\mathrm{Ca}^{2+}$ influx presumably through a mechanism involving the CAM/CAMKII axis (Tano and Vazquez 2011). A particularly interesting observation derived from those studies was that inhibition of PI3K function completely abrogated TNFa-dependent NFkB activation suggesting that the PI3K/AKT axis exerts a regulatory action on the NFkB pathway. Operation of such crosstalk has been demonstrated in cell types other than macrophages, where AKT-dependent transactivation of NFkB acts as an alternative AKT-dependent anti-apoptotic route independently of the AKT/BAD axis (Romashkova and Makarov 1999; Madrid, Wang et al. 2000; Bai, Ueno et al. 2009). In summary, our studies suggest that in human macrophages a CAM/CAMKII axis links constitutive $\mathrm{Ca}^{2+}$ influx to activation of $\mathrm{AKT}$, which then serves as a signaling node to promote survival through NFKB and/or phosphorylation of BAD.

Macrophages from both human and mouse origin express TRPC3, and TRPC3 constitutive function has been shown to be operational in different cell types from these two mammalian species. These attributes make TRPC 3 a great candidate to mediate the constitutive $\mathrm{Ca}^{2+}$ influx that supports the macrophage survival mechanisms described above. We indeed examined this possibility in recent studies that made use of bone-marrow derived macrophages obtained from mice globally deficient in TRPC3 (Tano, Smedlund et al. 2011). Macrophages derived from TRPC3 deficient bone marrow (TRPC3 $/-$ ) exhibited a significant reduction in constitutive cation influx compared to TRPC $3^{+/+}$cells (Tano, Smedlund et al. 2011). Most importantly, the number of apoptotic macrophages in response to TNFa was significantly higher in TRPC $3 /$ - cultures than in those of $\mathrm{TRPC}^{+/+}$macrophages, indicating a diminished survival in macrophages lacking TRPC3. Importantly, these observations correlated very well with the activation status of survival signaling: the phosphorylation of IkBa, AKT and BAD was severely reduced in TRPC3 $/-$ macrophages (Tano, Smedlund et al. 2011). Altogether, these findings indicated that TRPC3 has an obligatory role in macrophage survival and that TRPC3 is likely to mediate the constitutive $\mathrm{Ca}^{2+}$ influx required for proper operation of survival signaling.

As described above, clearance of apoptotic macrophages by resident phagocytes at the lesion site is fundamental for appropriate inflammation resolution, and is a major factor in determining lesion cellularity. By means of an in vitro efferocytosis assay in which $\mathrm{TRPC}^{+/+}$ and TRPC3\%- were used as either phagocytes or apoptotic cells, we observed that the phagocytic function of TRPC3-/-macrophages is drastically impaired when compared to that of $\mathrm{TRPC}^{+/+}$phagocytes; interestingly, apoptotic TRPC $3 \%$ cells seem to be poor substrates for phagocytosis regardless of the phagocyte's TRPC 3 expression status (Tano, Smedlund et al. 2011). Although additional studies are required to clarify TRPC3's role in efferocytosis, these findings suggest a critical requirement for TRPC3 within the signaling associated to phagocytic activity and/or cell-cell recognition processes that underlie efferocytosis. 


\section{TRPC3 as a prospective target in atherosclerosis: Roadmap of an exciting TRiP}

The rapid advance in elucidating signaling mechanisms associated to atherogenesis was enthusiastically perceived as an opportunity to develop anti-inflammatory strategies to manage the disease. However, the multifactorial nature of atherosclerosis makes such therapeutic strategies, often aimed at interfering on single targets, of limited efficacy and it is likely that multiple targeting is necessary to achieve clinically significant outcomes (Yonekawa and Harlan 2005; Preiss and Sattar 2007; Recio-Mayoral, Kaski et al. 2007). This is not surprising if we take in consideration the multifactorial nature of atherosclerosis and the diverse repertoire of signaling molecules and cell types that contribute to its pathogenesis. In that context, identifying new components of signaling events linked to monocyte recruitment and/or modulation of macrophage survival/apoptosis at the lesion site is of fundamental importance to move forward in the search for additional potential targets that could make those alternative therapeutic strategies a reality. Furthering our knowledge on the potential new roles of TRPC3, as well as other TRPC members, in atherogenesis can make a significant contribution to the search for new targets for the disease. Although ubiquitously expressed throughout tissues, TRPC3 on endothelial or macrophage surface could be used as a molecular target of relatively easy access for therapeutic and/or diagnostic purposes or may be exploited as a marker in non-invasive imaging, as it has been applied to other cell surface proteins (Kaufmann, Carr et al.; Saraste, Nekolla et al. 2009). The potential advantages of TRPC3 vs. traditional channel blockers as a prospective target for atherosclerosis has recently been discussed by us (Vazquez 2011). The exploration of TRPC3 as an atherorelevant signaling molecule is at its infancy, and several additional studies will be required to determine the impact of TRPC 3 expression/function on atherorelevant events. The generation and characterization of new mouse models of atherosclerosis with genetically manipulated levels of TRPC3 in the atherorelevant cell or tissue of interest (i.e., conditional knockouts or transgenics) will be a unique contribution to that goal.

\section{References}

Abramowitz, J. and L. Birnbaumer (2009). "Physiology and pathophysiology of canonical transient receptor potential channels." FASEB J. 23(2): 297-328.

Allen, S., S. Khan, et al. (1998). "Expression of adhesion molecules by Lp(a): a potential novel mechanism for its atherogenicity." FASEB J. 12(15): 1765-1776.

Arai, S., J. M. Shelton, et al. (2005). "A role for the apoptosis inhibitory factor AIM/Sp[alpha]/Api6 in atherosclerosis development." Cell metabolism 1(3): 201213.

Armesilla, A. L., E. Lorenzo, et al. (1999). "Vascular Endothelial Growth Factor Activates Nuclear Factor of Activated T Cells in Human Endothelial Cells: a Role for Tissue Factor Gene Expression." Mol. Cell. Biol. 19(3): 2032-2043.

Babaev, V. R., J. D. Chew, et al. (2008). "Macrophage EP4 Deficiency Increases Apoptosis and Suppresses Early Atherosclerosis." Cell metabolism 8(6): 492-501. 
Bai, D., L. Ueno, et al. (2009). "Akt-mediated regulation of NFkB and the essentialness of NFKB for the oncogenicity of PI3K and Akt." International Journal of Cancer 125(12): 2863-2870.

Chen, N. X., K. D. O'Neill, et al. (2002). "Signal transduction of \&\#x03B2;2m-induced expression of VCAM-1 and COX-2 in synovial fibroblasts." Kidney International 61(2): 414-424.

Chuluyan, E. H., L. Osborn, et al. (1995). "Domains 1 and 4 of vascular cell adhesion molecule-1 (CD106) both support very late activation antigen-4 (CD49d/CD29)dependent monocyte transendothelial migration." J Immunol 155: 3135-3144.

Cook-Mills, J. (2002). "VCAM-1 signals during lymphocyte migration: role of reactive oxygen species " Mol Immunol 39: 499-508.

Cook-Mills, J., J. Johnson, et al. (2004). "Calcium mobilization and Rac1 activation are required for VCAM-1 (vascular cell adhesion molecule-1) stimulation of NADPH oxidase activity." Biochem J 378: 539-547.

Cybulsky, M. I., K. Iiyama, et al. (2001). "A major role for VCAM-1, but not ICAM-1, in early atherosclerosis." J. Clin. Invest. 107(10): 1255-1262.

Dansky, H. M., C. B. Barlow, et al. (2001). "Adhesion of Monocytes to Arterial Endothelium and Initiation of Atherosclerosis Are Critically Dependent on Vascular Cell Adhesion Molecule-1 Gene Dosage." Arterioscler Thromb Vasc Biol 21(10): 16621667.

Datta, S. R., A. Brunet, et al. (1999). "Cellular survival: a play in three Akts." Genes \& Development 13(22): 2905-2927.

Deem, T. L., H. Abdala-Valencia, et al. (2007). "VCAM-1 Activation of Endothelial Cell Protein Tyrosine Phosphatase 1B." J Immunol 178(6): 3865-3873.

Galkina, E. and K. Ley (2007). "Vascular Adhesion Molecules in Atherosclerosis." Arterioscler Thromb Vasc Biol: 107.149179.

Hansson, G. K. (2005). "Inflammation, Atherosclerosis, and Coronary Artery Disease." N Engl J Med 352(16): 1685-1695.

Iiyama, K., L. Hajra, et al. (1999). "Patterns of Vascular Cell Adhesion Molecule-1 and Intercellular Adhesion Molecule-1 Expression in Rabbit and Mouse Atherosclerotic Lesions and at Sites Predisposed to Lesion Formation." Circ Res 85(2): 199-207.

Isabelle Ricard, M. D. P. G. D. (1997). "Clustering the adhesion molecules VLA-4 (CD49d/CD29) in Jurkat T cells or VCAM-1 (CD106) in endothelial (ECV 304) cells activates the phosphoinositide pathway and triggers Ca mobilization." European Journal of Immunology 27(6): 1530-1538.

Kaufmann, B. A., C. L. Carr, et al. Molecular Imaging of the Initial Inflammatory Response in Atherosclerosis: Implications for Early Detection of Disease. [Miscellaneous Article], Arteriosclerosis, Thrombosis \& Vascular Biology January 2010;30(1):5459.

Kim, I., S.-O. Moon, et al. (2001). "Vascular Endothelial Growth Factor Expression of Intercellular Adhesion Molecule 1 (ICAM-1), Vascular Cell Adhesion Molecule 1 (VCAM-1), and E-selectin through Nuclear Factor-kappa B Activation in Endothelial Cells." J. Biol. Chem. 276(10): 7614-7620. 
Linton, M. F., V. R. Babaev, et al. (1999). "A Direct Role for the Macrophage Low Density Lipoprotein Receptor in Atherosclerotic Lesion Formation." J. Biol. Chem. 274(27): 19204-19210.

Linton, M. F. and S. Fazio (2003). "Macrophages, inflammation, and atherosclerosis." Int J Obes Relat Metab Disord 27(S3): S35-S40.

Liu, J., D. P. Thewke, et al. (2005). "Reduced Macrophage Apoptosis Is Associated With Accelerated Atherosclerosis in Low-Density Lipoprotein Receptor-Null Mice." Arterioscler Thromb Vasc Biol 25(1): 174-179.

Madrid, L. V., C.-Y. Wang, et al. (2000). "Akt Suppresses Apoptosis by Stimulating the Transactivation Potential of the RelA/p65 Subunit of NF-kappa B." Mol. Cell. Biol. 20(5): 1626-1638.

Matheny, H. E., T. L. Deem, et al. (2000). "Lymphocyte Migration Through Monolayers of Endothelial Cell Lines Involves VCAM-1 Signaling Via Endothelial Cell NADPH Oxidase" J Immunol 164(12): 6550-6559.

Preiss, D. J. and N. Sattar (2007). "Vascular cell adhesion molecule-1: a viable therapeutic target for atherosclerosis?" International Journal of Clinical Practice 61(4): 697-701.

Quinlan, K. L., S. M. Naik, et al. (1999). "Substance P Activates Coincident NF-AT- and NF\{kappa\}B-Dependent Adhesion Molecule Gene Expression in Microvascular Endothelial Cells Through Intracellular Calcium Mobilization" J Immunol 163(10): 5656-5665.

Recio-Mayoral, A., J. C. Kaski, et al. (2007). "Clinical Trials Update from the European Society of Cardiology Congress in Vienna, 2007: PROSPECT, EVEREST, ARISE, ALOFT, FINESSE, Prague-8, CARESS in MI and ACUITY." Cardiovascular Drugs and Therapy 21(6): 459-465.

Romashkova, J. A. and S. S. Makarov (1999). "NF-[kappa]B is a target of AKT in antiapoptotic PDGF signalling." Nature 401(6748): 86-90.

Saraste, A., S. G. Nekolla, et al. (2009). "Cardiovascular molecular imaging: an overview." Cardiovasc Res 83(4): 643-652.

Seimon, T. A., Y. Wang, et al. (2009). "Macrophage deficiency of p381ิ \pm MAPK promotes apoptosis and plaque necrosis in advanced atherosclerotic lesions in mice." The Journal of Clinical Investigation 119(4): 886-898.

Seye, C. I., N. Yu, et al. (2003). "The P2Y2 Nucleotide Receptor Mediates UTP-induced Vascular Cell Adhesion Molecule-1 Expression in Coronary Artery Endothelial Cells." J. Biol. Chem. 278(27): 24960-24965.

Smedlund, K., J.-Y. Tano, et al. (2010). "The Constitutive Function of Native TRPC3 Channels Modulates Vascular Cell Adhesion Molecule-1 Expression in Coronary Endothelial Cells Through Nuclear Factor \{kappa\}B Signaling." Circ Res 106(9): 1479-1488.

Smedlund, K. and G. Vazquez (2008). "Involvement of Native TRPC3 Proteins in ATPDependent Expression of VCAM-1 and Monocyte Adherence in Coronary Artery Endothelial Cells." Arterioscler Thromb Vasc Biol 28(11): 2049-2055.

Smyth, J. T., W. I. DeHaven, et al. (2006). "Emerging perspectives in store-operated Ca2+ entry: Roles of Orai, Stim and TRP." Biochimica et Biophysica Acta (BBA) Molecular Cell Research 1763(11): 1147-1160. 
Tabas, I. (2010). "Macrophage death and defective inflammation resolution in atherosclerosis." Nat Rev Immunol 10(1): 36-46.

Tano, J.-Y., K. Smedlund, et al. (2011). "Impairment of survival signaling and efferocytosis in TRPC3-deficient macrophages." Biochemical and Biophysical Research Communications 410(3): 643-647.

Tano, J.-Y. and G. Vazquez (2011). "Requirement for non-regulated, constitutive calcium influx in macrophage survival signaling." Biochemical and Biophysical Research Communications 407(2): 432-437.

Tano, J. Y., K. Smedlund, et al. (2010). "Endothelial TRPC3/6/7 proteins at the edge of cardiovascular disease." Cardiovascular \& Hematological Agents in Medicinal Chemistry 8(1): 76-86.

Tergaonkar, V. (2006). "NF[kappa]B pathway: A good signaling paradigm and therapeutic target." The International Journal of Biochemistry \& Cell Biology 38(10): 16471653.

Trebak, M., G. Vazquez, et al. (2003). "The TRPC3/6/7 subfamily of cation channels." Cell Calcium 33(5-6): 451-61.

van Wetering, S., N. van den Berk, et al. (2003). "VCAM-1-mediated Rac signaling controls endothelial cell-cell contacts and leukocyte transmigration 10.1152/ajpcell.00048.2003." Am J Physiol Cell Physiol 285(2): C343-352.

Vazquez, G. (2011). "TRPC channels as prospective targets in atherosclerosis: terra incognita." Frontiers in Bioscience In press.

Vazquez, G. and J. W. Putney (2006). "Role of Canonical Transient Receptor Potential Channels (TRPC) in Receptor-Dependent Regulation of Vascular Cell Adhesion Molecule-1 In Human Coronary Artery Endothelium." Art. Thr. Vasc. Biol. 26: 9394.

Vazquez, G., B. J. Wedel, et al. (2004). "The mammalian TRPC cation channels." Biochimica et Biophysica Acta (BBA) - Molecular Cell Research 1742(1-3): 21-36.

Virmani, R., A. P. Burke, et al. (2006). "Pathology of the Vulnerable Plaque." Journal of the American College of Cardiology 47(8, Supplement 1): C13-C18.

Wang, Z., B. Liu, et al. (2008). "Phospholipase C Î23 deficiency leads to macrophage hypersensitivity to apoptotic induction and reduction of atherosclerosis in mice." The Journal of Clinical Investigation 118(1): 195-204.

Yancey, P. G., J. Blakemore, et al. (2010). "Macrophage LRP-1 Controls Plaque Cellularity by Regulating Efferocytosis and Akt Activation." Arterioscler Thromb Vasc Biol 30(4): 787-795.

Yao, Y.-G. and E. J. Duh (2004). "VEGF selectively induces Down syndrome critical region 1 gene expression in endothelial cells: a mechanism for feedback regulation of angiogenesis?" Biochemical and Biophysical Research Communications 321(3): 648656.

Yonekawa, K. and J. M. Harlan (2005). "Targeting leukocyte integrins in human diseases." J Leuk Biol 77(129-140).

Zhang, C. (2008). "The role of inflammatory cytokines in endothelial dysfunction." Basic Research in Cardiology 103(5): 398-406. 
Zola, H., B. Swart, et al. (2005). "CD molecules 2005: human cell differentiation molecules." Blood 106(9): 3123-3126. 


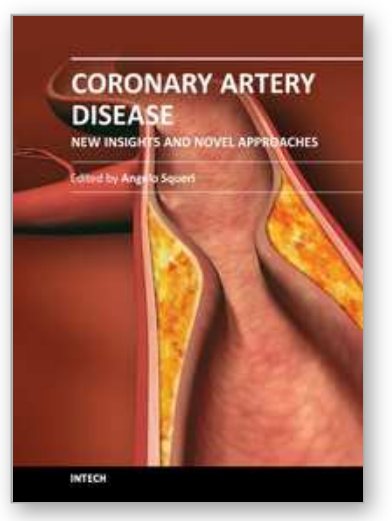

\author{
Coronary Artery Disease - New Insights and Novel Approaches \\ Edited by Dr. Angelo Squeri
}

ISBN 978-953-51-0344-8

Hard cover, 260 pages

Publisher InTech

Published online 16, March, 2012

Published in print edition March, 2012

Coronary Artery disease is one of the leading causes of death in industrialized countries and is responsible for one out of every six deaths in the United States. Remarkably, coronary artery disease is also largely preventable. The biggest challenge in the next years is to reduce the incidence of coronary artery disease worldwide. A complete knowledge of the mechanisms responsible for the development of ischaemic heart disease is an essential prerequisite to a better management of this pathology improving prevention and therapy. This book has been written with the intention of providing new concepts about coronary artery disease pathogenesis that may link various aspects of the disease, going beyond the traditional risk factors.

\title{
How to reference
}

In order to correctly reference this scholarly work, feel free to copy and paste the following:

Guillermo Vazquez, Kathryn Smedlund, Jean-Yves K. Tano and Robert Lee (2012). Molecular and Cellular Aspects of Atherosclerosis: Emerging Roles of TRPC Channels, Coronary Artery Disease - New Insights and Novel Approaches, Dr. Angelo Squeri (Ed.), ISBN: 978-953-51-0344-8, InTech, Available from:

http://www.intechopen.com/books/coronary-artery-disease-new-insights-and-novel-approaches/molecularand-cellular-aspects-of-atherosclerosis-emerging-roles-of-trpc-channels

\section{INTECH}

open science | open minds

\section{InTech Europe}

University Campus STeP Ri

Slavka Krautzeka 83/A

51000 Rijeka, Croatia

Phone: +385 (51) 770447

Fax: +385 (51) 686166

www.intechopen.com

\section{InTech China}

Unit 405, Office Block, Hotel Equatorial Shanghai

No.65, Yan An Road (West), Shanghai, 200040, China 中国上海市延安西路65号上海国际贵都大饭店办公楼 405 单元

Phone: +86-21-62489820

Fax: +86-21-62489821 
(C) 2012 The Author(s). Licensee IntechOpen. This is an open access article distributed under the terms of the Creative Commons Attribution 3.0 License, which permits unrestricted use, distribution, and reproduction in any medium, provided the original work is properly cited. 